University of New Orleans

ScholarWorks@UNO

3-1975

\title{
Ellipsometric function of a film-substrate system: Applications to the design of reflection-type optical devices and to ellipsometry
}

\author{
R. M.A. Azzam \\ University of New Orleans, razzam@uno.edu
}

A.-R. M. Zaghloul

N. M. Bashara

Follow this and additional works at: https://scholarworks.uno.edu/ee_facpubs

Part of the Electrical and Electronics Commons, and the Physics Commons

\section{Recommended Citation}

R. M. A. Azzam, A.-R. M. Zaghloul, and N. M. Bashara, "Ellipsometric function of a film-substrate system: Applications to the design of reflection-type optical devices and to ellipsometry," J. Opt. Soc. Am. 65, 252-260 (1975)

This Article is brought to you for free and open access by the Department of Electrical Engineering at ScholarWorks@UNO. It has been accepted for inclusion in Electrical Engineering Faculty Publications by an authorized administrator of ScholarWorks@UNO. For more information, please contact scholarworks@uno.edu. 


\title{
Ellipsometric function of a film-substrate system: Applications to the design of reflection-type optical devices and to ellipsometry*
}

\author{
R. M. A. Azzam, ${ }^{\dagger}$ A.-R. M. Zaghloul, and N. M. Bashara \\ Electrical Materials Laboratory-College of Engineering, University of Nebraska, Lincoln, Nebraska 68508 \\ (Received 16 September 1974) \\ The ratio $\rho=R_{p} / R_{s}$ of the complex amplitude-reflection coefficients $R_{p}$ and $R_{s}$ for light polarized \\ parallel $(p)$ and perpendicular $(s)$ to the plane of incidence, reflected from an optically isotropic \\ film-substrate system, is investigated as a function of the angle of incidence $\phi$ and the film thickness \\ $d$. Both constant-angle-of-incidence contours (CAIC) and constant-thickness contours (CTC) of the \\ ellipsometric function $\rho(\phi, d)$ in the complex $\rho$ plane are examined. For transparent films, $\rho(\phi, d)$ is \\ a periodic function of $d$ with period $D_{\phi}$ that is a function of $\phi$. For a given angle of incidence $\phi$ \\ and film thickness $d\left(0 \leq \phi \leq 90,0 \leq d<D_{\phi}\right)$, the equispaced linear array of points \\ $\left(\phi, d+m D_{\phi}\right)(m=0,1,2, \ldots)$ in the real $(\phi, d)$ plane is mapped by the periodic function $\rho(\phi, d)$ \\ into one distinct point in the complex $\rho$ plane. Conversely, for a given film-substrate system, any \\ value of the ellipsometric function $\rho$ can be realized at one particular angle of incidence $\phi$ and an \\ associated infinite series of film thicknesses $d, d+D_{\phi}, d+2 D_{\phi}, \ldots$. This analysis leads to (1) a \\ unified scheme for the design of all reflection-type optical devices, such as polarizers and retarders, \\ (2) a novel null ellipsometer without a compensator, for the measurement of films whose thicknesses \\ are within certain permissible ranges, and (3) an inversion procedure for the nonlinear equations of \\ reflection ellipsometry that separates the determination of the optical constants (refractive indices \\ and extinction coefficients) of the film and substrate from the film thickness. Extension of the \\ work to absorbing films is discussed.
}

Index Headings: Ellipsometry; Reflection; Polarization; Films.

We consider the case of light reflection by a substrate covered by a single film. As shown in Fig. 1, the film of thickness $d$ has parallel plane boundaries and is sandwiched between semi-infinite ambient and substrate media. The ambient (medium 0), the film (medium 1), and the substrate (medium 2) are all homogeneous and optically isotropic with refractive indices $N_{0}, N_{1}$, and $N_{2}$, respectively. The complex amplitude-reflection coefficients $R_{p}$ and $R_{s}$ for light polarized with its electric vector vibrating parallel $(p)$ and perpendicular $(s)$ to the plane of incidence are given by

$$
R_{\nu}=\frac{r_{01 \nu}+r_{12 \nu} e^{-j 2 \beta}}{1+r_{01 \nu} r_{12 \nu} e^{-j 2 \beta}}, \nu=p, s
$$

where $r_{01 \nu}$ and $r_{12 \nu}$ are the ambient-film $(0-1)$ and film-substrate $(1-2)$ interface Fresnel reflection coefficients for the two fundamental polarizations, $\nu=p$ and $\nu=s$. The film phase thickness $\beta$ is given by

$$
\beta=2 \pi(d / \lambda)\left(N_{1}^{2}-N_{0}^{2} \sin ^{2} \phi_{0}\right)^{1 / 2},
$$

where $\lambda$ is the free-space wavelength of light and $\phi_{0}$ is the angle of incidence in medium 0 . The ellipsometric function $\rho$ is defined as the ratio ${ }^{1,2}$

$$
\rho=R_{p} / R_{s} \text {, }
$$

of the $p$ and $s$ complex amplitude-reflection coefficients. The ellipsometric function $\rho$ is usually written as

$$
\rho=\tan \psi e^{j \Delta},
$$

where $\tan \psi$ is the $p$-to-s relative amplitude attenuation and $\Delta$ is the $p$-to-s relative phase shift upon reflection. From Eqs. (1) and (3), we obtain

$$
\rho=\frac{r_{01 \beta}+r_{12 \beta} e^{-j 2 \beta}}{1+r_{01 \rho} r_{12 \beta} e^{-j 2 \beta}} \times \frac{1+r_{01 s} r_{12 s} e^{-j 2 \beta}}{r_{01 s}+r_{12 s} e^{-j 2 \beta}} .
$$

The purpose of this paper is to study the behavior of the ellipsometric function $\rho$ for a given ambient-film- substrate system with known refractive indices $N_{0}, N_{1}$, and $N_{2}$ as a function of its two remaining arguments, the angle of incidence $\phi\left(=\phi_{0}\right)$ and the film thickness $d$. The formulation provides a unified framework for the design of all reflection-type film-substrate optical devices, such as polarizers and retarders. In addition, the analysis of this paper leads to a novel null ellipsometer without a compensator that is applicable, in general, when the film thickness is of the order of a wavelength or more. It also points the way to an inversion procedure of the nonlinear equations of reflection ellipsometry that promises to be efficient, rapidly convergent, and accurate.

For simplicity, we assume the ambient to be air $\left(N_{0}=1\right)$, which is usually the case. We initially consider transparent films and later discuss the effect of absorption. Also we take the $\mathrm{Si}-\mathrm{SiO}_{2}$ system, which is technologically important, as an example and assume the light source to be the widely available $6328 \AA \mathrm{He}-\mathrm{Ne}$ laser. At $\lambda=6328 \AA$, the refractive indices of $\mathrm{SiO}_{2}$ and Si are 1.46 and $3.85-j 0.02$, respectively. ${ }^{3}$

0

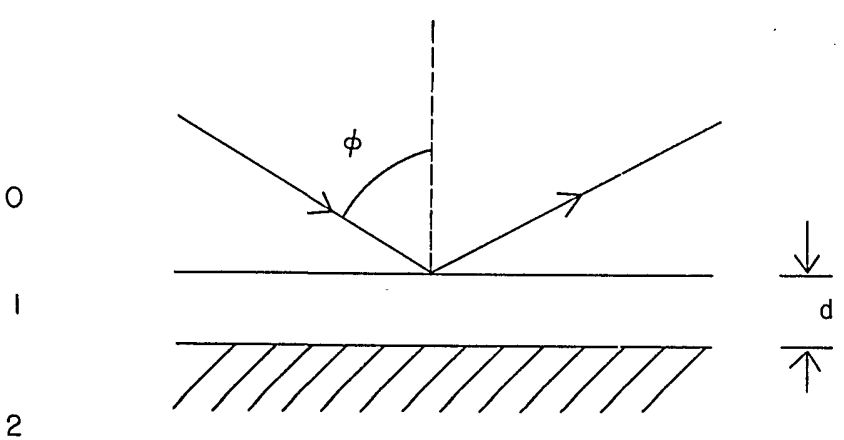

।

FIG. 1. Film-substrate system. Medium 0 is the ambient, 1 is the film, and 2 is the substrate. 


\section{CONSTANT-ANGLE-OF-INCIDENCE CONTOURS (CAIC) OF THE ELLIPSOMETRIC FUNCTION $\rho(\phi, d)$}

Equations (1) can be cast in the form

$$
R_{p}=\frac{a+b X}{1+a b X}, \quad R_{s}=\frac{c+d X}{1+c d X},
$$

and subsequently Eq. (5) becomes

$$
\rho=\frac{(a+b X)(1+c d X)}{(1+a b X)(c+d X)},
$$

or

$$
\rho=\frac{A+B X+C X^{2}}{D+E X+F X^{2}} .
$$

In Eqs. (6)-(8), we have

$$
\begin{aligned}
& X=e^{-j 2 \beta}, \\
& (a, b)=\left(r_{01 p}, r_{12 p}\right),(c, d)=\left(r_{01}, r_{12 s}\right),
\end{aligned}
$$

and

$$
\begin{array}{lll}
A=a, & B=(b+a c d), & C=b c d, \\
D=c, & E=(d+a b c), & F=a b d .
\end{array}
$$

Equations (6) show that each of the complex amplitudereflection coefficients $R_{p}$ and $R_{s}$ is related to the complex exponential function of film thickness $X$, Eq. (9), by a bilinear transformation with coefficients determined by the 0-1 and 1-2 interface Fresnel reflection coefficients, ${ }^{4}$ Eqs. (10). Equation (8) shows that the ratio of the $p$ and $s$ reflection coefficients $\rho$ is a rational function of $X$ in the form of a quadratic divided by another quadratic with coefficients determined by the interface Fresnel reflection coefficients, Eqs. (10) and (11).

If we substitute the film phase thickness $\beta$ from Eq. (2) into Eq. (9) and set $N_{0}=1$ and $\phi_{0}=\phi$, the complex exponential function $X$ becomes

$$
X=\exp \left[-j 4 \pi(d / \lambda)\left(N_{1}^{2}-\sin ^{2} \phi\right)^{1 / 2}\right],
$$

or

$$
X=\exp \left[-j 2 \pi\left(d / D_{\phi}\right)\right],
$$

where

$$
D_{\phi}=\frac{\lambda}{2}\left(N_{1}^{2}-\sin ^{2} \phi\right)^{-1 / 2} .
$$
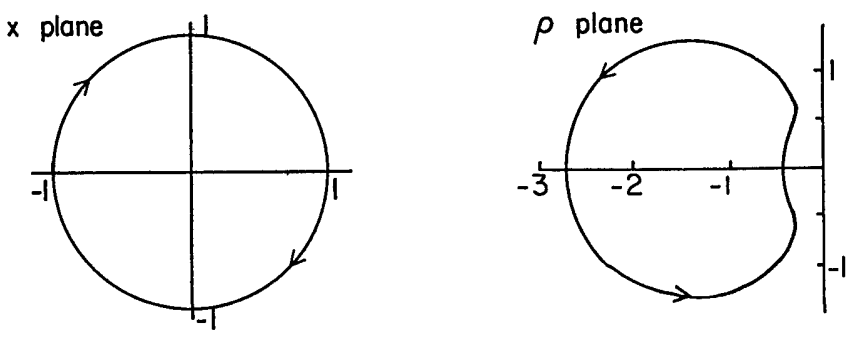

FIG. 2. Left: The unit circle in the complex $X$ plane. Right: Constant-angle-of-incidence contour (CAIC) of the ellipsometric function $\rho$ at an angle of incidence $\phi=60^{\circ}$ for $\mathrm{Si}-\mathrm{SiO}_{2}$ system at a wavelength $\lambda=6328 \AA$.

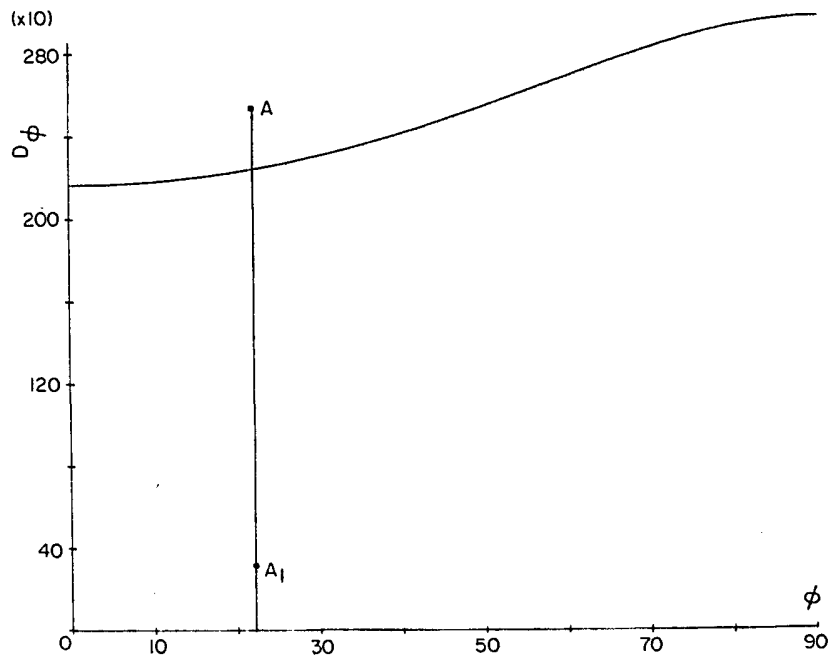

FIG. 3. Thickness period $D_{\phi}$ in angstroms for $\mathrm{SiO}_{2}$ film $\left(N_{1}\right.$ $=1.46)$ at $\lambda=6328 \AA$ as a function of the angle of incidence $\phi$, where $\phi$ is in degrees. Point $A \equiv(\phi, d)$ can be brought down vertically, at the same $\phi$, into point $A_{1} \equiv\left(\phi, d-D_{\phi}\right)$ that realizes the same ellipsometric function $\rho$ as point $A$, at a different film thickness $\left(d-D_{\phi}\right)$.

For transparent films, $N_{1}$ and $D_{\phi}$ are real. In this case, at any angle of incidence $\phi$, it can be seen from Eq. (12) that the representative point of the complex exponential function $X$ moves uniformly in a clockwise direction around the unit circle in the complex $X$ plane, as the film thickness $d$ is increased, starting from the point $X=1$ when $d=0$, Fig. 2 (left). Note that $X$ is a periodic function of $d$, so that the unit circle is cyclically traversed. The first full revolution around the unit circle is completed as the film thickness sweeps the interval or period

$$
0 \leqslant d<D_{\phi} \text {. }
$$

The thickness period $D_{\phi}$, Eq. (13), is a function of the angle of incidence $\phi$ that is completely determined by the wavelength $\lambda$ and the film refractive index $N_{1}$. Figure 3 shows a plot of the thickness period $D_{\phi}$ versus the angle of incidence $\phi$ when $\lambda=6328 \AA$ and $N_{1}=1.46$, which corresponds to the $\mathrm{SiO}_{2}$ film. The minimum value of $D_{\phi}$ occurs at $\phi=0^{\circ}, D_{\phi}=2167.13 \AA$, whereas the maximum value occurs at $\phi=90^{\circ}, D_{\phi}=2974.34 \AA$.

Because the ellipsometric function $\rho$ is related to the complex exponential $X$ by an analytic rational function of $X$, Eq. (8), the representative point of $\rho$ must trace a closed contour in the complex $\rho$ plane as $X$ traces the unit circle in the complex $X$ plane (when the film thickness $d$ is increased at a given angle of incidence $\phi$ ). Figure 2 (right) shows such a constant-angle-of -incidence contour ${ }^{5}$ (CAIC) when $\phi=60^{\circ}$ for the $\mathrm{Si}-\mathrm{SiO}_{2}$ system at $\lambda=6328 \AA$. As $\phi$ scans its entire range

$$
0 \leqslant \phi \leqslant 90^{\circ} \text {, }
$$

the coefficients of the rational function $\rho(X)$, and the period $D_{\phi}$, change so that a whole family of nonintersecting constant $-\phi$ (variable $-d$ ) contours is generated to fill the entire space of the complex $\rho$ plane. Figure 4 shows such a family for the $\mathrm{Si}-\mathrm{SiO}_{2}$ system at $\lambda=6328 \AA$. The 


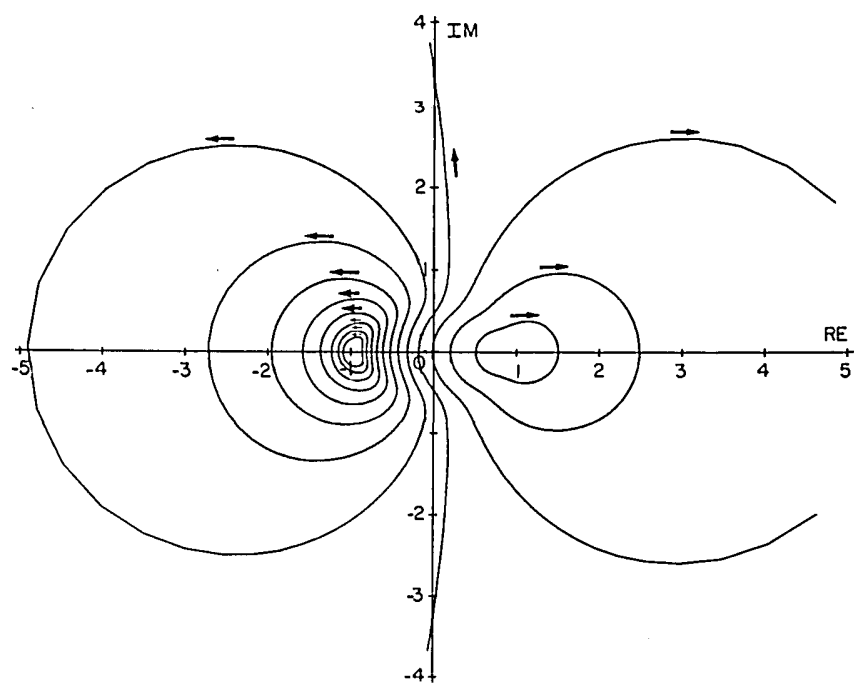

FIG. 4. Constant-angle-of-incidence contours (CAIC's) in the complex $\rho$ plane for $\mathrm{Si}-\mathrm{SiO}_{2}$ system at $\lambda=6328 \AA$ starting near $\rho=-1$ at an angle of incidence $\phi=30^{\circ}$ with a step of $5^{\circ}$. On each contour, the arrow indicates the direction in which the film thickness increases.

$\phi=0^{\circ}$ (normal incidence) and $\phi=90^{\circ}$ (grazing incidence) contours collapse to the null points $\rho=-1$ and $\rho=+1$ on the real axis because, at these angles, $R_{p}=-R_{s}$ and $R_{b}=R_{s}$, respectively, for any film thickness. All CAIC's that correspond to angles of incidence in a certain range

$$
0<\phi<\phi_{s},
$$

enclose the point $\rho=-1$, whereas the remaining contours that correspond to

$$
\phi_{s}<\phi<90^{\circ} \text {, }
$$

enclose the point $\rho=+1$. The direction in which the thickness increases is indicated by an arrow on each contour. Arrows on all CAIC's enclosing $\rho=-1$ point the same way and opposite to the arrows on all the other contours enclosing $\rho=+1$. The angle of incidence $\phi_{s}$ that appears in inequalities (16) leads to a CAIC that passes through the point at infinity. This means that, at this angle, a value (or a set of values equally spaced by the period $D_{\phi_{s}}$ ) of film thickness can be found such that $\rho=\infty$. Under such conditions, the film-substrate sys tem acts as an $s \backsim$ suppressing reflection polarizer. The parameters of such a polarizer will be obtained in Sec. III. The locus of points on the CAIC's where the film thickness is zero, $d=0$, is the same as the locus of

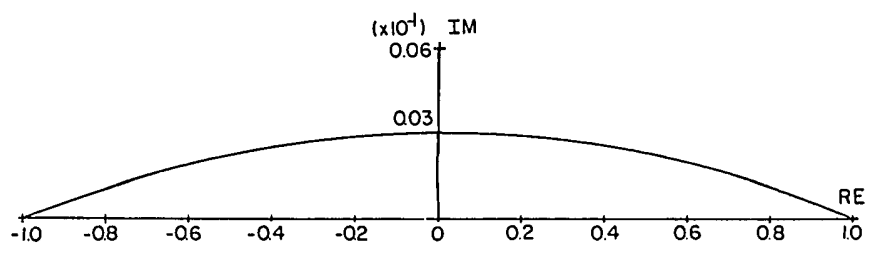

FIG. 5. Zero-thickness contour (ZTC) in the complex $\rho$ plane for $\mathrm{Si}-\mathrm{SiO}_{2}$ system at $\lambda=6328 \AA$.

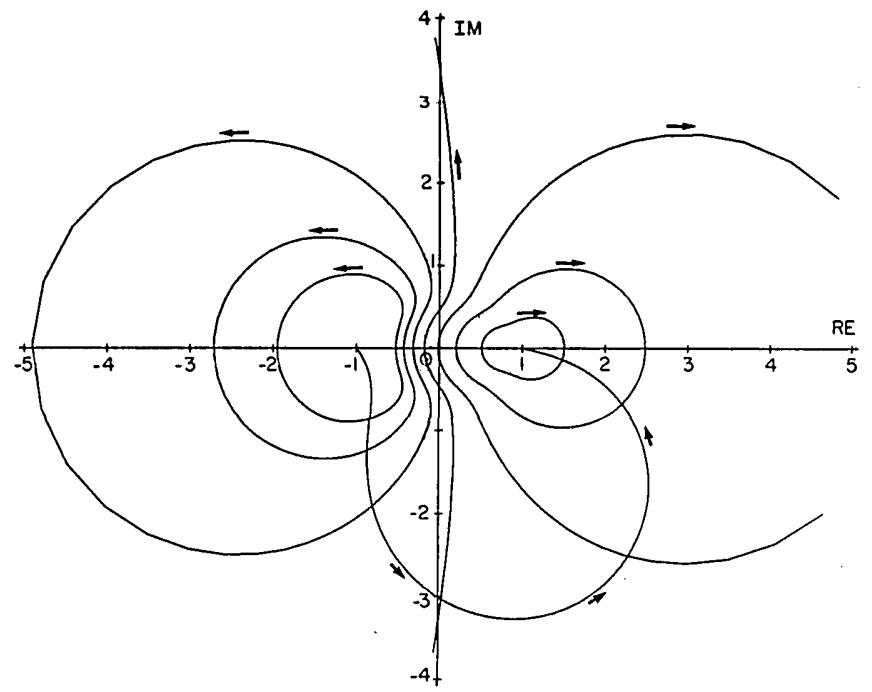

FIG. 6. A constant-thickness contour (CTC) for a film thickness $d=0.24 \lambda \AA$ superimposed on the constant-angle-of-incidence contours of $\phi=55^{\circ}$ to $\phi=85^{\circ}$, with a step of $5^{\circ}$, in the complex $\rho$ plane for $\mathrm{Si}-\mathrm{SiO}_{2}$ system at $\lambda=6328 \AA$.

$$
\rho=\rho_{02}=r_{02 \phi} / r_{02 s},
$$

which is the ratio of the Fresnel reflection coefficients of the ambient-substrate interface. Because of the low absorption of Si at $6328 \AA$, the bare-substrate zerothickness contour (ZTC) is almost coincident with the straight-line segment from $\rho=-1$ to $\rho=+1$ through the origin. This contour is shown on an expanded scale in Fig. 5 .

\section{CONSTANT-THICKNESS CONTOURS (CTC) OF THE ELLIPSOMETRIC FUNCTION $\rho(\phi, d)$}

The bare-substrate zero-thickness contour, which is shown in Fig. 5, is one member of a complementary family of constant-thickness contours (CTC) that can be drawn in the complex $\rho$ plane. For any given value of film thickness $d$, the associated CTC can be generated from the zero-thickness contour (ZTC) with the help of

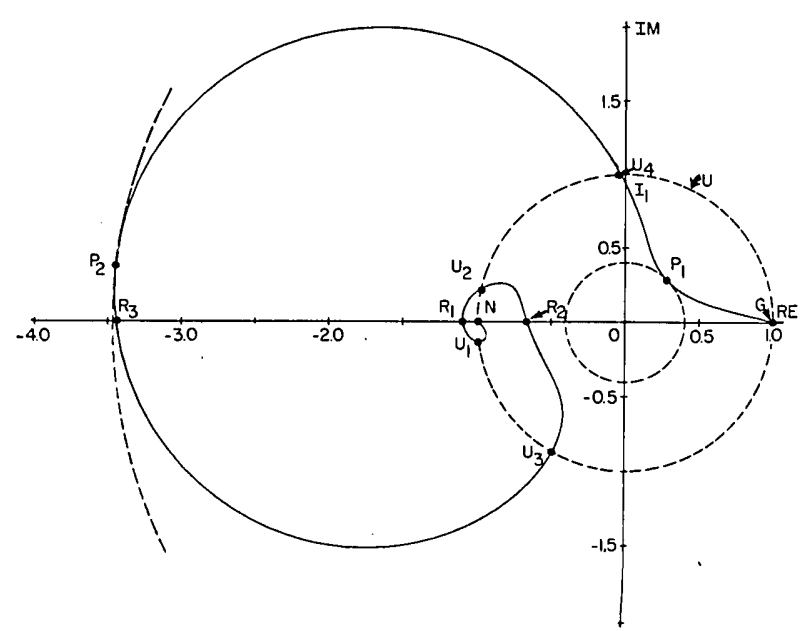

FIG. 7. Constant-thickness contour (CTC) in the complex $\rho$ plane for $\mathrm{Si}-\mathrm{SiO}_{2}$ system at $\lambda=6328 \AA$ and film thickness $d$ $=1.5 \mu \mathrm{m}$. 


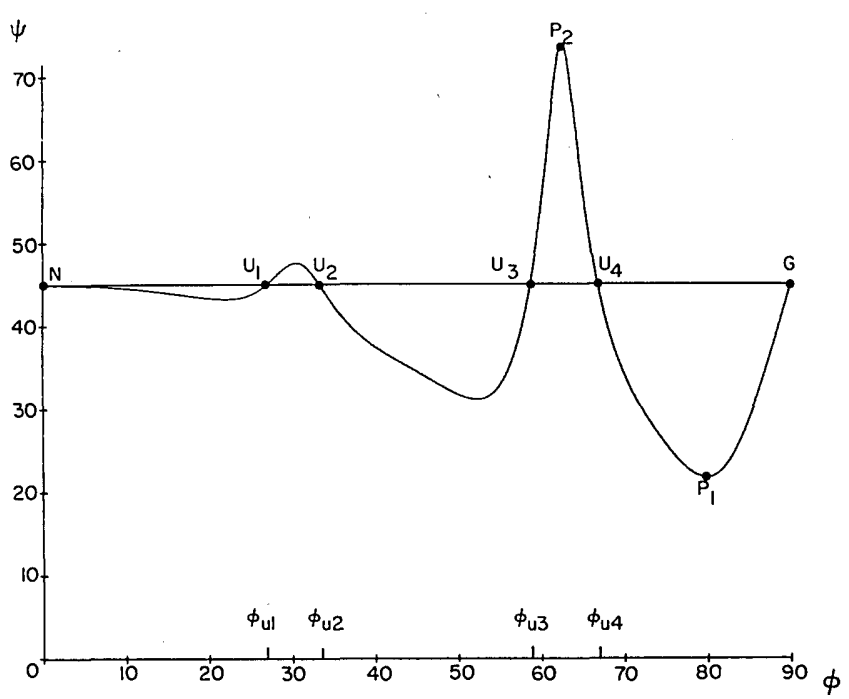

FIG. 8. The angle $\psi$ as a function of the angle of incidence $\phi$ for $\mathrm{Si}-\mathrm{SiO}_{2}$ system at $\lambda=6328 \AA$ and film thickness $d=1.5 \mu \mathrm{m}$. Both $\psi$ and $\phi$ are in degrees.

the constant-angle-of-incidence contours (CAIC) of Fig. 4. Each point on the ZTC corresponds to one angle of incidence $\phi$ and, as $\phi$ is increased from $\phi=0^{\circ}$ to $\phi=90^{\circ}$, we move along the ZTC once from $\rho=-1$ to $\rho=+1$. At a given angle of incidence $\phi$, a point on the $\mathrm{ZTC}$ can be moved around the constant $\phi$ contour to a new point that corresponds to the thickness $d$. Joining such points, for the same value of film thickness $d$, at different values of $\phi$, generates the constant-thickness contour, Fig. 6. The points on the ZTC at $\phi=0^{\circ}$ and $\phi=90^{\circ}$, which coincide with $\rho=-1$ and $\rho=+1$, respectively, stay the same for any value of $d$.

Figure 7 shows the $d=1.5 \mu \mathrm{m}$ constant-thickness contour of the ellipsometric function $\rho$ in the complex $\rho$ plane for the $\mathrm{Si}-\mathrm{SiO}_{2}$ system at $\lambda=6328 \AA$. This contour is traversed as the angle of incidence $\phi$ is increased starting from the point $N(\rho=-1)$ when $\phi=0^{\circ}$ (normal incidence) and ending at the point $G(\rho=+1)$ when $\phi=90^{\circ}$ (grazing incidence). The spiralling behavior near $N$ shown by the constant-thickness contour of Fig. 7 is shared by other such contours for thicknesses comparable to or greater than $\lambda$. This spiralling can be attributed to the high density of the CAIC's near $N$ (see Fig. 4 ) and to the fact that the thickness period $D_{\phi}$ of the CAIC is shortest (hence the rate of rotation is maximum) near $\phi=0^{\circ}$ (see Fig. 3).

The angles $\psi$ and $\Delta$ associated with the ellipsometric function $\rho=\tan \psi e^{j \Delta}[\mathrm{Eq}$. (4)] are plotted in Figs. 8 and 9 versus the angle of incidence $\phi$ as we move along the $1.5 \mu \mathrm{m}$ constant-thickness contour of Fig. 7. Several significant points are marked on the CTC of Fig. 7 and on the $\psi$ and $\Delta$ curves in Figs. 8 and 9 , respectively. With the exception of $N$ and $G$, these significant points are grouped as follows:

(i) The intersection points $R_{1}, R_{2}, \ldots$ of the CTC with the real axis of the complex $\rho$ plane, which occur at angles of incidence $\phi_{r 1}, \phi_{r 2} \ldots$ : The intersection $R_{i}$ of the
CTC with the real axis is to the left or to the right of the origin if $\Delta=\pi$ or 0 , respectively. All intersections $R_{1}$, $R_{2}$, and $R_{3}$ for the $d=1.5 \mu \mathrm{m} \mathrm{CTC} \mathrm{shown} \mathrm{in} \mathrm{Fig.} 7$ correspond to $\Delta=\pi$ and occur at angles of incidence $\phi_{r 1}=30.13^{\circ}, \phi_{r 2}=46.75^{\circ}$, and $\phi_{r 3}=62.44^{\circ}$, respectively. Such points appear in Fig. 9 as the intersections of the $\Delta-\phi$ curve with the $\Delta=\pi$ line. When $\Delta=0$ or $\pi$, incident light that is linearly polarized at any azimuth relative to the plane of incidence is linearly polarized after it is reflected from the film-coated substrate. This suggests a new polarizer-surface-analyzer (PSA) null-ellipsometer arrangement without a compensator that can be used to characterize film-substrate systems for which the film thickness exceeds a certain minimum value so that one or more intersections of the CTC with the real axis become available. This minimum thickness, for the same system, depends on the wavelength; the smaller the wavelength used, the smaller the minimum thickness. Three such intersections are obtained at an oxide thickness of $1.5 \mu \mathrm{m}$ in the case of the $\mathrm{Si}-\mathrm{SiO}_{2}$ system at $6328 \AA$, Fig. 7. The nulling scheme would involve setting either the polarizer $P$ or the analyzer $A$ at a fixed azimuth and adjusting the other element ( $A$ or $P$, respectively), together with the angle of incidence $\phi$, for null. The null condition can be obtained at as many angles of incidence $\phi_{r k}$ as there are intersections $R_{k}$ of the CTC with the real axis, where $k=1,2, \ldots$ Experimental measurements using this PSA null-ellipsometer arrangement were carried out on $\mathrm{Si}-\mathrm{SiO}_{2}$ system at a wavelength of $6328 \AA$ for different film thicknesses. The results obtained were quite close to the calculated values using the nominal film thickness and the published data for the optical constants ${ }^{3}$ of $\mathrm{Si}$ and $\mathrm{SiO}_{2}$. Details of this novel method of null ellipsometry will be considered elsewhere.

(ii) The intersections $I_{1}, I_{2}, \ldots$ of the CTC with the imaginary axis of the complex $\rho$ plane, which occur at angles of incidence $\phi_{i 1}, \phi_{i 2}, \ldots$ : At such points, $\Delta=\pi / 2$ or $3 \pi / 2$, dependent on whether the intersection $I$ is above or below the origin, respectively. When $\Delta=\pi / 2$ or $3 \pi / 2$, the major and minor axes of the ellipse of polarization of the reflected light, when the incident light is linearly polarized at an arbitrary azimuth, are

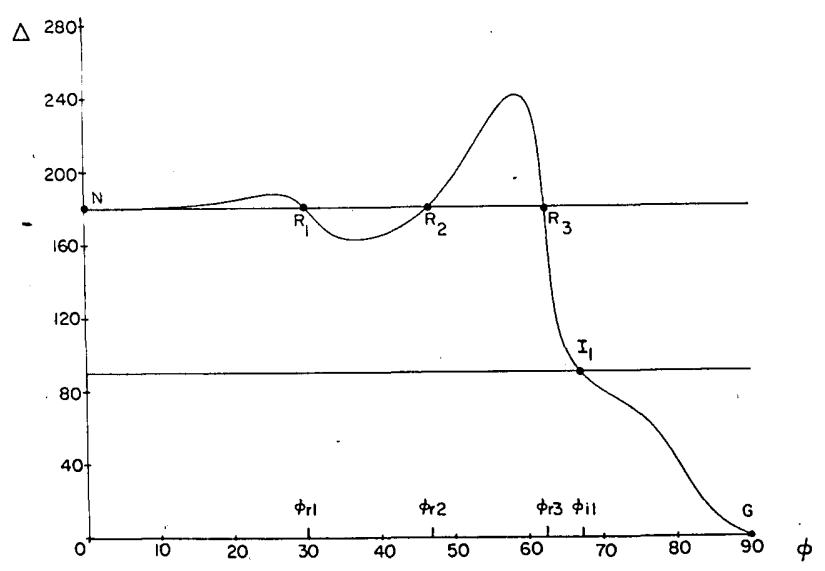

FIG. 9. The angle $\Delta$ as a function of the angle of incidence $\phi$ for $\mathrm{Si}-\mathrm{SiO}_{2}$ system at $\lambda=6328 \AA$ and film thickness $d=1.5 \mu \mathrm{m}$. Both $\Delta$ and $\phi$ are in degrees. 

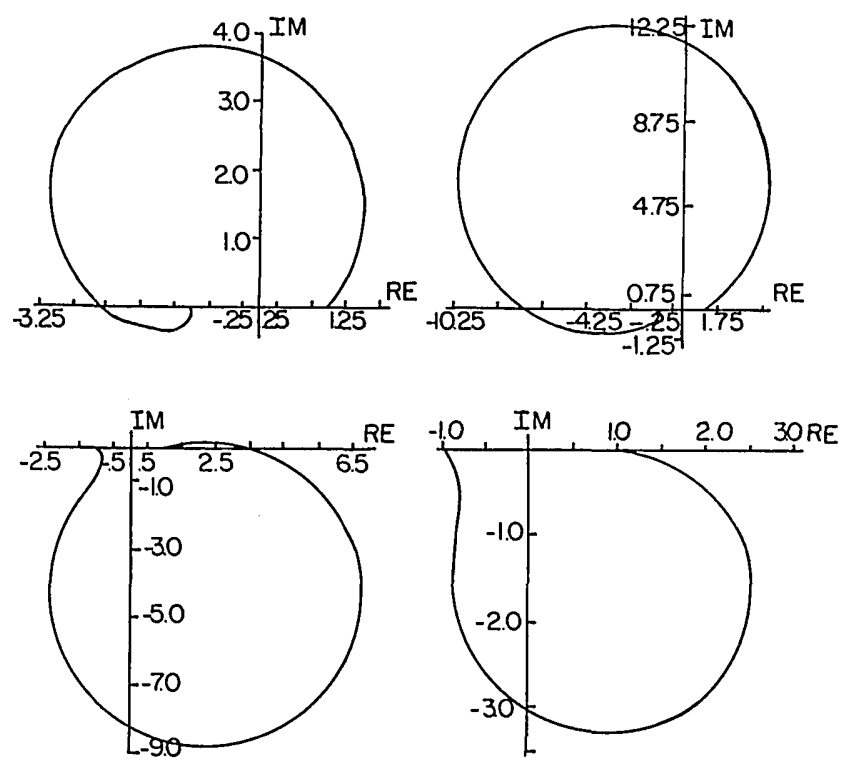

FIG. 10. A sequence of constant-thickness contours (CTC's) in the complex $\rho$ plane for the $\mathrm{Si}-\mathrm{SiO}_{2}$ system at $\lambda=6328 \AA$ for different film thicknesses. Upper left: $d=0.21 \lambda$; upper right: $d=0.22 \lambda$; lower left: $d=0.23 \lambda$; and lower right: $d=0.24 \lambda$.

aligned parallel and perpendicular to the plane of incidence. The angles of incidence $\phi_{i 1}, \phi_{i 2}, \ldots$ may be called the principal angles ${ }^{6}$ of incidence for the filmsubstrate system. For the $d=1.5 \mu \mathrm{m} \mathrm{CTC} \mathrm{of} \mathrm{Fig.} \mathrm{7,}$ only one principal angle is available $\phi_{i 1}=67.19^{\circ}$. The $I$ intersections appear in Fig. 9 as the intersections of the $\Delta$-vs $-\phi$ curve with the $\Delta=\pi / 2$ line.

(iii) The intersection points $U_{1}, U_{2}, \ldots$ of the CTC with the unit circle $U$ of the complex $\rho$ plane: At such intersections, we have $\tan \psi=1$; hence $\psi=45^{\circ}$. These points are shown in Fig. 8 as the intersections of the $\psi$-vs- $\phi$ curve with the $\psi=45^{\circ}$ line. When $\tan \psi=1$, we have $\left|R_{p}\right|$ $=\left|R_{s}\right|$ and $\rho=e^{j \Delta}[$ see Eqs. (3) and (4)], and the film-substrate system acts as a reflection retarder. Such a retarder exhibits isotropic absorption because the absolute reflectances $R=\left|R_{p}\right|^{2}=\left|R_{s}\right|^{2}$ are necessarily less than 1 . For the $\mathrm{Si}-\mathrm{SiO}_{2}$ system with $d=1.5 \mu \mathrm{m}$ at $\lambda=6328 \AA$, the angles of incidence at which exact operation as a retarder is achieved are $\phi_{u 1}=27.0^{\circ}, \phi_{u 2}=33.25^{\circ}, \phi_{u 3}=58.75^{\circ}$, and $\phi_{u 4}=66.75^{\circ}$. The associated retardations are $\Delta_{u 1}=187.62^{\circ}, \Delta_{u 2}=167.23^{\circ}, \Delta_{u 3}=240.9^{\circ}$, and $\Delta_{u 4}=92.03^{\circ}$, whereas the reflectances are $R_{u 1}=0.105$, $\mathfrak{R}_{u 2}=0.108, \mathfrak{R}_{u 3}=0.155$, and $R_{u 4}=0.208$, respectively. A more systematic approach to the design of retarders is discussed in Sec. III.

(iv) The points $P_{1}$ and $P_{2}$ where the minimum-radius and maximum-radius circles, centered on the origin, touch the CTC, respectively: These two concentric circles define an annular domain in which the CTC is confined. At $P_{1}$ and $P_{2},|\rho|=\tan \psi$ is minimum and maximum, respectively. These points indicate how a given film-substrate system comes close to operating as a $p$ - or $s$-suppressing polarizer. When the minimum radius is zero, $\psi_{P_{1}}=0$, the CTC passes through the origin. At the corresponding angle of incidence $\phi_{P_{1}}$ the filmsubstrate system acts exactly as a $p$-suppressing polar- izer. On the other hand, when the maximum radius becomes infinitely large, $\psi_{P 2}=90^{\circ}$, the CTC passes through the point at infinity. At the corresponding angle of incidence $\phi_{P_{2}}$, the film-substrate system acts exactly as an $s$-suppressing polarizer. The basic design of such elements will also be discussed in Sec. III.

Figures 10 and 11 show two sequences of constantthickness contours (CTC). As can be appreciated from these sequences, there is an infinite variety of such CTC's. Two CTC's may intersect one another, so that a given value of the ellipsometric function $\rho$ can be realized at two different thicknesses at the same angle of incidence. ${ }^{7}$ This multiplicity is absent from the constant$\phi$ contours, each of which is traced only once in the interval of film thickness $0 \leqslant d<D_{\phi}$, Eq. (14), where $D_{\phi}$ is given by Eq. (13) and shown in Fig. 3 for $\mathrm{SiO}_{2}$ at $\lambda=6328 \AA$. By limiting the choice of thickness at each $\phi$ to the range specified by Eq. (14), i. e., by limiting ourselves to the area below the $D_{\phi}$ boundary curve in the $(\phi, d)$ space of Fig. 3, all values of the ellipsometric function $\rho$ are generated only once. Notice that any $(\phi, d)$ point in Fig. 3 above the $D_{\phi}$ boundary curve can be brought vertically down (i.e., at the same $\phi$ ) by subtraction of the appropriate multiple of the period $D_{\phi}$. Recall that the ellipsometric function $\rho$ [Eqs. (8) and (12)] is periodic in film thickness $d$, with an angle-ofincidence-dependent period $D_{\phi}$.

\section{DESIGN OF REFLECTION-TYPE FILM-SUBSTRATE OPTICAL DEVICES}

We have seen that by varying the angle of incidence $\phi$ over the interval $0 \leqslant \phi \leqslant 90^{\circ}$, and varying the film thickness $d$ at each angle $\phi$ over the thickness period $0 \leqslant d<D_{\phi}$ (Fig. 3), we cover the entirety of the complex $\rho$ plane of the ellipsometric function $\rho$. In other words, a thickness value $d$ and an angle of incidence $\phi$ can always be found that realize any given value of $\rho$, i. e., any given values of $\psi$ and $\Delta$. This assumes a film-substrate system with zero absorption in the film. Because
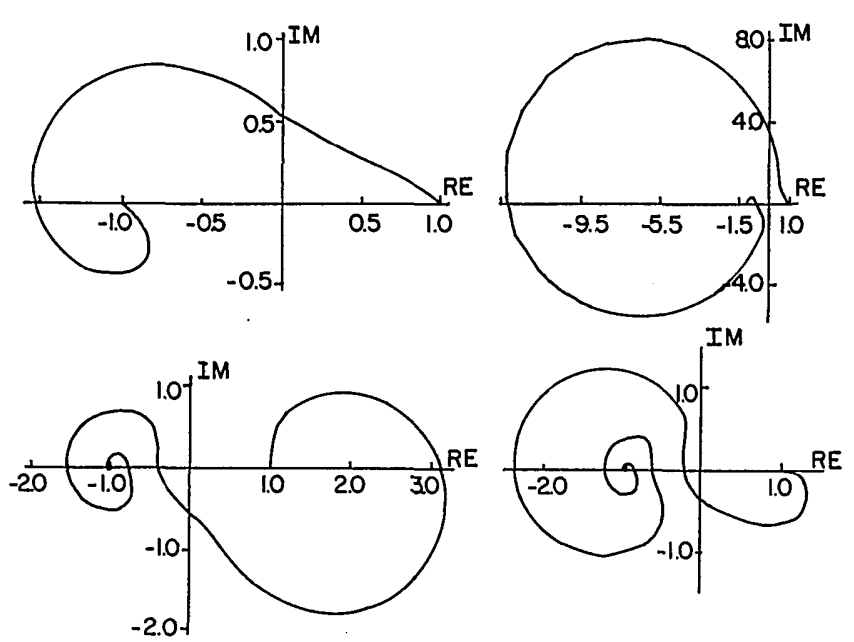

FIG. 11. A sequence of constant-thickness contours (CTC's) in the complex $\rho$ plane for the $\mathrm{Si}-\mathrm{SiO}_{2}$ system at $\lambda=6328 \AA$ for different film thicknesses. Upper left: $d=\lambda$; upper right: $d=2 \lambda$; lower left: $d=3 \lambda$; and lower right: $d=4 \lambda$. 
$\rho$ determines completely the effect of reflection on the state of polarization of the incident light, determination of $d$ and $\phi$ that lead to a prescribed value of $\rho$ (or $\psi$ and $\Delta)$ represents the main step in the design of film-substrate reflection optical devices.

\section{A. Reflection polarizers}

The ellipsometric function $\rho=R_{p} / R_{s}$ is a rational function of the complex exponential $X, E q$. (8), that has one zero, $\rho=0$, when

$$
R_{p}=0 \text {, }
$$

and one pole, $\rho=\infty$, when

$$
R_{s}=0 \text {. }
$$

Because $\rho$ is the ratio of two quadratics, it has one additional pole and one additional ze ro plus those specified by Eqs. (18) and (19). They correspond to $R_{p}=\infty$ and $R_{s}=\infty$, respectively, and, hence, are of no physical meaning.

The condition $R_{p}=0$ leads to a simple solution of $\mathrm{Eq}$. (8) for $X$, namely [see Eqs. (1) and (6)]

$$
\begin{aligned}
& r_{01 p}+r_{12 p} X=0, \\
& X=-\left(r_{01 p} / r_{12 p}\right) .
\end{aligned}
$$

Because, for transparent films [Eqs. (12) and (13)],

$$
|X|=1 \text {, }
$$

a very simple procedure for the design of a film-substrate, $p$-suppressing, reflection polarizer becomes quite evident. The right-hand side of Eq. (20) is the ratio of the 0-1 and 1-2 interface Fresnel reflection coefficients for the $p$ polarization, which are independent of film thickness and vary only with angle of incidence for a given film-substrate system. Thus, by plotting $\left|r_{01 p} / r_{12 p}\right| \equiv|X|$ with the angle of incidence $\phi$, the intersection of such a curve with the straight line $|X|=1$ yields the polarizing angle of incidence at which suppression of the parallel polarization is possible by a film-substrate system. Figure 12 (upper left) shows a curve $^{8}$ of $1-|X|$ vs $\phi$. Such a curve intersects the $\phi$ axis at the polarizing angle $\phi_{p}=75.4401^{\circ}$. To determine the necessary film thickness for polarizer operation, let us write the complex Fresnel coefficients $r_{01 p}$ and $r_{12 p}$ as

$$
r_{01 p}=\left|r_{01 p}\right| e^{j 601 \phi}, \quad r_{12 \phi}=\left|r_{12 \phi}\right| e^{j 6} 12 \phi \text {, }
$$

where $\delta_{01 p}$ and $\delta_{12 p}$ are the interface phase jumps. By substituting $r_{01 p}$ and $r_{12 p}$ from Eqs. (22) and $X$ from Eq. (12) into $\mathrm{Eq}$. (20), we obtain

$$
-e^{-j 2 \pi\left(d / D_{\phi}\right)}=\left(\left|r_{01 p}\right| /\left|r_{12 p}\right|\right) e^{j\left(\delta_{01 p}-\delta_{12 p}\right)} \text {. }
$$

At the $p$-polarizing angle $\phi_{p},\left|r_{01 p}\right|=\left|r_{12 p}\right|$, (this is how $\phi_{p}$ was found), so that Eq. (23a) becomes

$$
-e^{-j 2 \pi\left(d_{\phi} / D_{\phi p}\right)}=e^{j\left(\sigma_{01 p}-\delta_{12 p}\right) \phi \phi} .
$$

$D_{\phi_{\phi}}$ and $\left(\delta_{01 \phi}-\delta_{12 p}\right)_{\phi p}$ are the values of $D_{\phi}$ and $\left(\delta_{01 p}-\delta_{12 \phi}\right)$ evaluated at $\phi=\phi_{p}$. Equation (23b) can be immediately
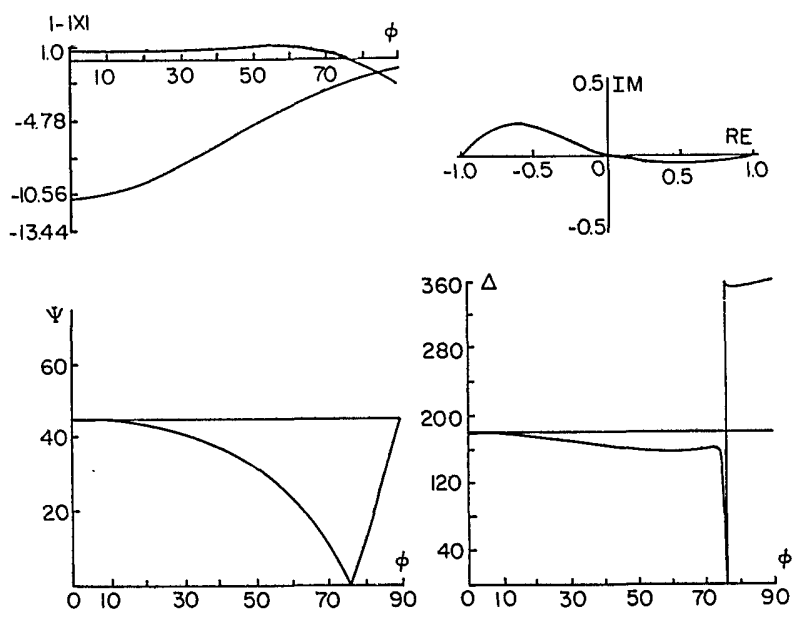

FIG. 12. This group of curves represent the design and performance of the $p$-suppressing polarizer for the $\mathrm{Si}-\mathrm{SiO}_{2}$ system at $\lambda=6328 \AA$. Upper left: The quantity $1-|X|$, where $X$ is the thickness complex-exponential function, plotted against the angle of incidence $\phi$, where $\phi$ is in degrees. Upper right: The constant-thickness contour (CTC) for film thickness $d$ $=2891.73 \AA$. Lower left: The angle $\psi$ as a function of the angle of incidence $\phi$ for the same film thickness. Both $\psi$ and $\phi$ are in degrees. Lower right: The angle $\Delta$ as a function of the angle of incidence $\phi$ for the same film thickness. Both $\Delta$ and $\phi$ are in degrees.

solved for the $p$-polarizing film thickness $d_{p}$,

$$
\begin{aligned}
& -2 \pi\left(d_{p} / D_{\phi_{p}}\right)+2 \pi\left(m+\frac{1}{2}\right)=\left(\delta_{01 p}-\delta_{12 p}\right)_{\phi_{p}}, \\
& \left(d_{p} / D_{\phi_{p}}\right)=\frac{1}{2 \pi}\left(\delta_{12 p}-\delta_{01 p}\right)_{\phi_{p}}+\left(m+\frac{1}{2}\right),
\end{aligned}
$$

or

$$
d_{p}=\frac{1}{2 \pi}\left(\delta_{12 p}-\delta_{01 p}\right)_{\phi_{p}} D_{\phi_{p}}+\left(m+\frac{1}{2}\right) D_{\phi_{p}} .
$$

In Eq. (24), the appearance of the multiple $m D_{\phi_{p}}$ of the thickness period $D_{\phi_{p}}(m$ is an integer) is consistent with the fact that $\rho$ is a periodic function of film thickness with a period equal to $D_{\phi_{p}}$ when $\phi=\phi_{p}$. For the $\mathrm{Si}-\mathrm{SiO}_{2}$ system, the least-polarizing thickness was found to be $d_{p}=2891.73 \AA$. . The thickness period $D_{\phi_{p}}$ at $\phi=\phi_{p}$ was found to be $2894.61 \AA$. Thus, the sequence of polarizing thicknesses in angstroms is $2891.73,5786.34,8680.95$, ..., which are equispaced by the thickness period $D_{\phi_{p}}$. The constant-thickness contour (CTC) at a polarizing thickness of $2891.73 \AA$ is shown in Fig. 12 (upper right). It passes through the origin $(\rho=0)$, as it should. The associated $\psi-\phi$ and $\Delta-\phi$ curves at that same thickness are shown in Fig. 12 (lower left and lower right, respectively). Notice that $\psi$ drops to zero at the polarizing angle, Fig. 12 (lower left), and that $\Delta$ experiences a discontinuous jump of $2 \pi$ at that angle, Fig. 12 (lower right). The calculated $p$ and $s$ reflectances, $R_{p_{p}}$ and $R_{s_{p}}$, of this $p$-suppressing polarizer were found to be $0.23 \times 10^{-4}$ and 0.76 , respectively.

The design of an $s$-suppressing polarizer follows exactly the same procedure that we outlined for the design of the p-suppressing polarizer. All we have to do is rewrite Eqs. (20)-(24) with an $s$ subscript instead of a $p$ 
subscript. Our calculations for the $\mathrm{Si}-\mathrm{SiO}_{2}$ system at $6328 \AA$ show that the $s$-polarizing angle $\phi_{s_{p}}=70.56^{\circ}$ and that the least-polarlaing thickness $d_{s}=1417.69 \AA$. The thickness period $D_{\phi_{s}}$ at $\phi_{s}$ is equal to $2838.65 \AA$. Thus the infinite sequence of $s$-polarizing film thicknesses in angstroms is $1417.69,4256.34,7094.99, \ldots$. Figure 13 (upper right) shows the CTC at $d_{s}=1417.69 \AA$ and Fig. 13 (lower left and lower right) give the associated $\psi-\phi$ and $\Delta-\phi$ curves. Note that the CTC passes through the point at infinity, Fig. 13 (upper right), that $\psi=90^{\circ}$ at $\phi=\phi_{s}$, Fig. 13 (lower left), and that $\Delta$ experiences a discontinuous jump of $\pi$ at $\phi_{s}$, Fig. 13 (lower right). The calculated $p$ and $s$ reflectances of the above $s$-suppressing polarizer, $R_{p_{s}}$ and $R_{s_{s}}$, are 0.28 and $0.19 \times 10^{-11}$, respectively.

The above approach to the design of reflection filmsubstrate polarizers is equivalent to that of RuizUrbieta et al. ${ }^{9-11}$ However, the analysis leading to the design equations is much simpler. The simplicity comes from working with the complex amplitude-reflection coefficients $R_{p, s}$ directly rather than the reflectances $R_{p, s}=\left|R_{p, s}\right|^{2}$ and the realization that for zero reflectance, $R_{p, s}=0$, the complex amplitude-reflection coefficient $R_{p, s}$ must itself be zero.

\section{B. Reflection retarders}

To realize any value of the ellipsometric function $\rho$, we can generally solve Eq. (8) for $X$ in terms of $\rho$

$$
X=\frac{-(B-\rho E) \pm\left[(B-\rho E)^{2}-4(C-\rho F)(A-\rho D)\right]^{1 / 2}}{2(C-\rho F)},
$$

where $A, B, C, D, E$, and $F$ are functions of the interface reflection coefficients $r_{01 p}, r_{01 s}, r_{12 p}$, and $r_{12 s}$ only, Eqs. (10) and (11). To determine the angle of incidence $\phi$ at which a given value of $\rho$ is realizable, we make use of the fact that $|X|=1$ and that the right-hand side of Eq. (25) is a function of $\phi$ only. Thus, by plotting $1-|X|$ vs $\phi$, the intersection of the resulting curve with the $\phi$ axis gives the desired value of $\phi$. Equation (25) predicts two branches for $1-|X|$ corresponding to the two complex roots. Only one of them can intersect the $\phi$ axis because of the nonintersecting nature of the constant-angle-of-incidence contours (CAIC), Fig. 4. The required film thickness together with the above-determined angle of incidence lead to the specified value of $\rho$. Let $\alpha$ be the angle of the complex number on the right-hand side of Eq. (25). Then

$$
-2 \pi\left(d / D_{\phi}\right)+m 2 \pi=\alpha,
$$

from which

$$
d=-\frac{\alpha}{2 \pi} D_{\phi}+m D_{\phi},
$$

where $\alpha$ and $D_{\phi}$ are evaluated at the previously determined $\phi$.

The design of any retarder is a special case of the foregoing general scheme. ${ }^{12}$ In this case
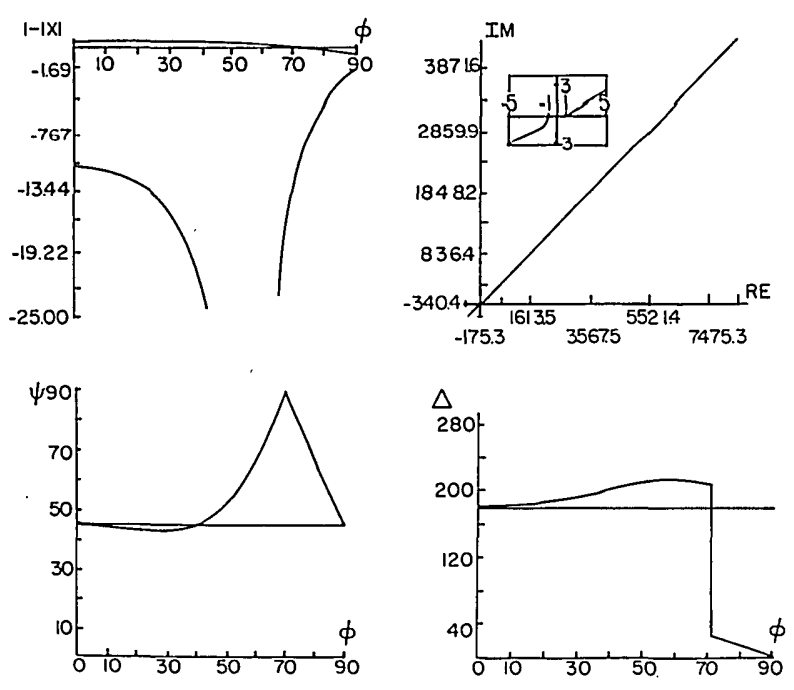

FIG. 13. This group of curves represent the design and performance of the $s$-suppressing polarizer for the $\mathrm{Si}-\mathrm{SiO}_{2}$ system at $\lambda=6328 \AA$. Upper left: The quantity $1-|X|$, where $X$ is the thickness complex-exponential function plotted against the angle of incidence $\phi$, where $\phi$ is in degrees. Upper right: The constant-thickness contour (CTC) for film thickness $d_{s}=1417.69$ $\AA$. The part of the curve around zero is shown in the box on a different scale. Lower left: The angle $\psi$ as a function of the angle of incidence $\phi$ for the same film thickness. Both $\psi$ and $\phi$ are in degrees. Lower right: The angle $\Delta$ as a function of the angle of incidence $\phi$ for the same film thickness. Both $\Delta$ and $\phi$ are in degrees.

$$
\rho=e^{j \Delta}
$$

where $\Delta$ is the desired retardation. For a quarter-wave retarder (QWR), $\Delta=\pi / 2$ and $\rho=+j$. By substituting this value of $\rho$ into Eq. (25), we calculated $1-\left|X_{1}\right|$ and $1-\left|X_{2}\right|$ vs $\phi$ for the $\mathrm{Si}-\mathrm{SiO}_{2}$ system at $6328 \AA$. The results are shown in Fig. 14 (upper left). Notice that only one branch $\left|X_{1}\right|$ intersects the $\phi$ axis. The angle of intersection $\phi_{\mathrm{QWR}}=67.29^{\circ}$. The smallest film thickness for operation as a quarter-wave retarder $d_{\mathrm{QWR}}=1056.29 \AA$, is obtained from Eq. (26) where $m=0$. The reflectances of this QWR are $\mathbb{R}_{p_{\mathrm{QWR}}}=\mathbb{R}_{s_{\mathrm{QWR}}}=0.215$. Figure 14 (upper right) shows the CTC corresponding to $d_{\mathrm{QWR}}$. Such a curve passes through the point $+j$ on the imaginary axis, as expected. Figure 14 (lower left) shows $\psi$ vs $\phi$ when $d=d_{\text {QWR }}$, while Fig. 14 (lower right) shows $\Delta$ vs $\phi$ for the same thickness. Note that $\psi$ stays quite close to the $\psi=+45^{\circ}$ line, leading to the very interesting conclusion that such a film-substrate system can operate as an angle-of-incidence tunable retarder. The tuning curve of such a retarder is Fig. 14 (lower right).

\section{INVERSION OF THE NONLINEAR EQUATIONS OF REFLECTION ELLIPSOMETRY}

From the above, we have seen that for transparent films any value of the ellipsometric function $\rho$ can be realized by solving Eq. (8) for $X$ in terms of $\rho$, Eq. (25). The coefficients $A, B, C, D, E$, and $F$ of Eq. (25) are functions of the angle of incidence $\phi$ and the optical 

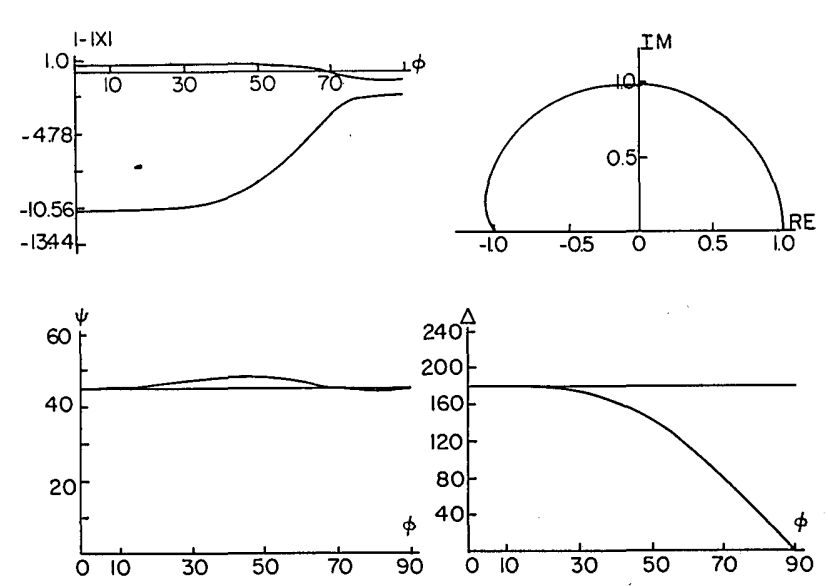

FIG. 14. This group of curves represent the design and performance of the quarter-wave-retarder for the $\mathrm{Si}-\mathrm{SiO}_{2}$ system at $\lambda=6328 \AA$. Upper left: The quantity $1-|X|$, where $X$ is the thickness complex-exponential function, plotted against the angle of incidence $\phi$, where $\phi$ is in degrees. Upper right: The constant-thickness contour (CTC) at film thickness $d_{\mathrm{QWR}}$ $=1056.29 \AA$. Lower left: The angle $\psi$ as a function of the angle of incidence $\phi$ for the same film thickness. Both $\psi$ and $\phi$ are in degrees. Lower right: The angle $\Delta$ as a function of the angle of incidence $\phi$ for the same film thickness. Both $\Delta$ and $\phi$ are in degrees.

constants (refractive indices and extinction coefficients) of the ambient, film, and substrate only, but not of the film thickness $d$. Also, we found that $X$ should satisfy the condition $|X|=1$, Eq. (21). Therefore, if the angle of incidence $\phi$, the wavelength $\lambda$, and the ellipsometric function $\rho$ are known, the optical constants of the film and the substrate should satisfy the condition $|X|=1$, Eq. (21), independently of the film thickness $d$. By reflection ellipsometry, we measure the ellipsometric function $\rho$ at a given angle of incidence $\phi$ and wavelength $\lambda$. The number of independent measurements necessary for the inversion procedure depends on the number of unknowns to be determined. We need one measurement for each pair of unknowns. To carry out the inversion process, we write the required equations, one from each measurement, in the form of $|X|=1$, where $X$ is given by Eq. (25). The required independent measurements can be made, for example, at multiple angles of incidence. The numerical-inversion procedure is to iterate on the optical constants of both the film and the substrate until the condition $|X|=1$, where $X$ is given by Eq. (25), is satisfied for all such equations. Substituting the optical constants thus obtained into Eq. (26), we obtain the film thickness. ${ }^{13}$

The foregoing outlines a unique method of separating the determination of the optical constants of the film and substrate from that of the film thickness. As a simple special case, a simulation study was carried out to determine the film refractive index $N_{1}$ and its thickness for $\mathrm{Si}-\mathrm{SiO}_{2}$ system at a wavelength of $6328 \AA$. The results confirmed the method outlined. An error of $0.1^{\circ}$ in the angle of incidence $\phi$ produces an error of 0.001 in the determination of the film refractive index $N_{1}$ of 1.46 and an error of $50 \AA$ in the determination of an assumed film thickness of $31640 \AA$.

Reinberg ${ }^{14}$ arrived at a method equivalent to ours for the special case of solving for the refractive index $N_{1}$ and thickness $d$ of a nonabsorbing film. However, our method can provide the solution for all of the parameters of a filmed substrate, that is, the refractive index. $N_{1}$ of a nonabsorbing film and $n_{2}$ and $k_{2}$, the refractive index and extinction coefficient of the substrate in addition to the film thickness $d$.

\section{v. EFFECT OF FILM ABSORPTION}

We close with a brief discussion of the effect of film absorption, which is generally applicable to either the design of the reflection-type optical devices or to the inversion procedure that we have discussed previously in Sec. III and IV, respectively. The concepts remain the same as for transparent films, but the condition of Eq. (21), $|X|=1$, is no longer satisfied. When the film is absorbing, the complex exponential function $X$, Eqs. (9), (12), and (13), traces a convergent logarithmic spiral in the complex $X$ plane, instead of the unit circle in the case of transparent films. Note that the exponential function of Eq. (12) contains a damping term in addition to the periodic term, because the film refractive index $N_{1}$ is a complex quantity. Because of this damping term, the procedure to find the angle of incidence $\phi$ and the film thickness $d$ that realize a given value of the ellipsometric function $\rho$ is changed.

Equation (25) continues to provide the solution for $X$ in terms of the ellipsometric function to be realized and the interface reflection coefficients $r_{01 p}, r_{01 s}, r_{12 p}$, and $r_{12 s}$ independent of the film thickness $d$. By taking the logarithms of both sides of Eq. (12), we obtain

$$
\ln (X)=-j 2 \pi\left(d / D_{\phi}\right),
$$

where $X$ is given by Eq. (25). From Eqs. (28) and (13), we get

$$
d=j(\lambda / 4 \pi)\left(N_{1}^{2}-\sin ^{2} \phi\right)^{-1 / 2} \ln (X)+\frac{1}{2} m \lambda\left(N_{1}^{2}-\sin ^{2} \phi\right)^{-1 / 2},
$$

where the second term has been added because $X$ and $X e^{j m 2 \pi}$ are indistinguishable, $m$ being a positive or negative integer.

Equation (29) is the basis of a procedure for obtaining the angle of incidence $\phi$ and the film thickness $d$ that realize the ellipsometric function $\rho$. For a given filmsubstrate system, at a given wavelength, the right-hand side of Eq. (29) is a complex function of the angle of incidence only. The left-hand side is always real and equals the film thickness. Thus, to get the solution of Eq. (29), the angle of incidence $\phi$ should satisfy the condition that the right-hand side of this equation is real. If we plot the right-hand side of the equation in the complex plane as a function of the angle of incidence $\phi$, the intersection of the resulting curve with the real axis gives the value of the film thickness $d$. The angle of incidence at which the intersection occurs gives the required angle of incidence. Note that we have to plot 
the right-hand side of Eq. (29) for each value of $m$ $(m=0, \pm 1, \pm 2, \ldots)$ leading to a number of possible solution sets for the film thickness $d$ and the angle of incidence $\phi$. As the film absorption increases, the number of discrete solution sets $\left(\phi_{m}, d_{m}\right), m=0, \pm 1, \ldots$ that realize the required $\rho$ are expected to become smaller until the required $\rho$ is realized at only one thickness and one angle of incidence, or not at all.

To determine the optical constants of both the film and the substrate $\left(n_{1}, k_{1} ; n_{2}, k_{2}\right)$ and the film thickness $d$ from multiple ellipsometric measurements, an adequate set of independent equations of the form of Eq. (29), to be obtained from the multiple measurements, is solved by iterating on the optical constants in a manner analogous to the procedure outlined in Sec. IV for nonabsorbing films.

*Work supported by the National Science Foundation.

$\dagger$ Also with the Division of Hematology, Department of Internal Medicine, College of Medicine, The University of Nebraska Medical Center, Omaha, Neb. 68105.

${ }^{1}$ Ellipsometry in the Measurement of Surfaces and Thin Films, edited by E. Passaglia, R. Stromberg, and J. Kruger, Natl. Bur. Std. (U.S.) Misc. Pub. 1, No. 256 (U. S. Government Printing Office, Washington, D. C., 1961).

${ }^{2}$ Proceedings of the Symposium on Recent Developments in Ellipsometry, edited by N. M. Bashara, A. B. Buckman, and A. C. Hall (North-Holland, Amsterdam, 1968).
${ }^{3}$ Ellipsometric Tables of the $\mathrm{Si}_{-} \mathrm{SiO}_{2}$ System for Mercury and $\mathrm{He}$-Ne Laser Spectral Lines, edited by G. Gergely (Akademiai Kiado, Budapest, 1971).

${ }^{4}$ The significance of this was appreciated by A. B. Winterbottom, Optical Studies of Metal Surfaces, Kgl. Norskē Vidensk. Selsk. Skrift., Vol. 1 (F. Bruns, Trondheim, 1955).

${ }^{5}$ Such contours are also called the polar curves. See the paper by P. C. S. Hayfield, Ref. 1, pp. $157 \mathrm{ff}$.

${ }^{6}$ The principal angle is defined for a bare substrate as that angle at which $\Delta=\pi / 2$.

${ }^{7}$ These thicknesses are spaced by an integral multiple of the thickness period $D_{\phi}$ evaluated at the common angle of incidence $\phi$.

${ }^{8}$ Two branches are shown in Fig. 12 (upper left). The lower branch is generated from the condition that $R_{s}=\infty$ which has no physical existence but leads to $\rho=0$. In this case, $x=-1 /$ $\boldsymbol{r}_{01 s} \boldsymbol{r}_{12 s}$, from which the lower branch is calculated. Both the upper and lower branches are predicted by the general design equation, Eq. (25), in which, for the present case of a $p$-suppressing reflection polarizer, $\rho$ is set equal to zero.

${ }^{9}$ M. Ruiz-Urbieta and E. M. Sparrow, J. Opt. Soc. Am. 62, 1188 (1972).

${ }^{10}$ M. Ruiz-Urbieta and E. M. Sparrow, J. Opt. Soc. Am. 63, 194 (1973).

${ }^{11}$ M. Ruiz-Urbieta, E. M. Sparrow, and G. W. Goldman, Appl. Opt. 12, 590 (1973).

${ }^{12}$ So also is the design of reflection polarizers. For the $p$-suppressing and $s$-suppressing reflection polarizers, the values of $\rho$ to be substituted in the right-hand side of Eq. (25) are 0 and $\infty$, respectively.

${ }^{13}$ This film thickness is the least one; to get the correct film thickness the appropriate multiple of $D_{\phi}$ should be added.

${ }^{14}$ A. R. Reinberg, Appl. Opt. 11, 1273 (1972). We want to thank a reviewer for calling this reference to our attention. 